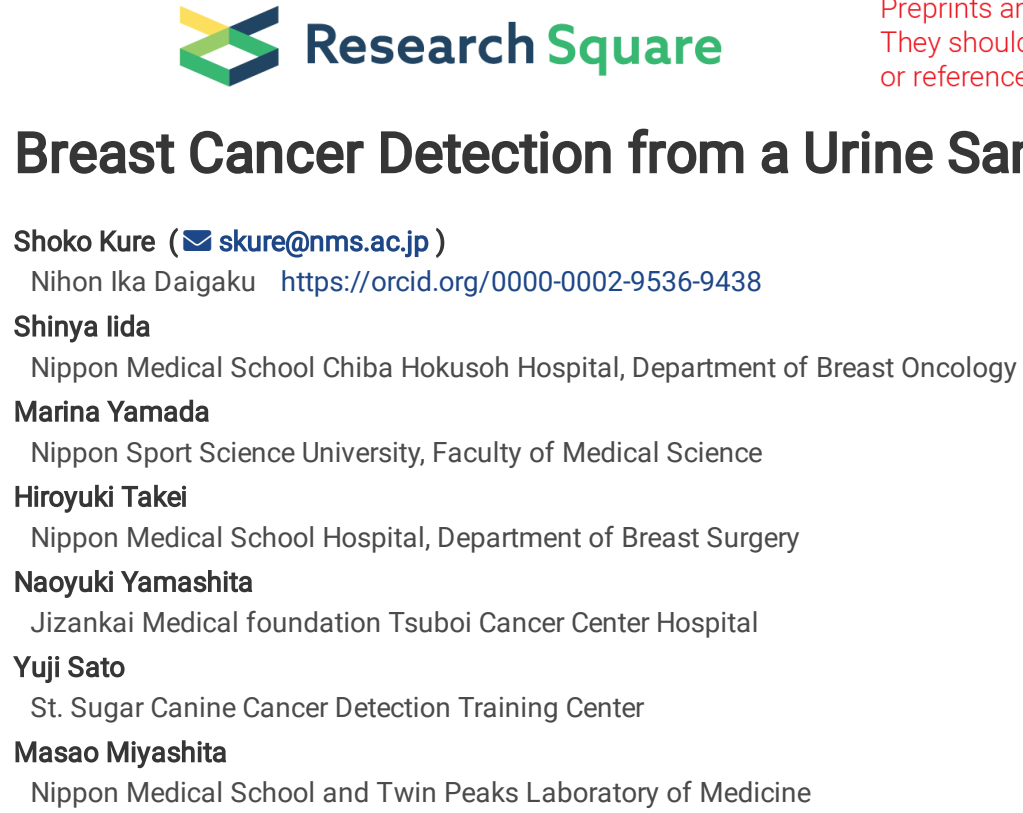

Shoko Kure ( $\nabla$ skure@nms.ac.jp)

Nihon lka Daigaku https://orcid.org/0000-0002-9536-9438

Shinya lida

Nippon Medical School Chiba Hokusoh Hospital, Department of Breast Oncology

Marina Yamada

Nippon Sport Science University, Faculty of Medical Science

Hiroyuki Takei

Nippon Medical School Hospital, Department of Breast Surgery

Naoyuki Yamashita

Jizankai Medical foundation Tsuboi Cancer Center Hospital

Yuji Sato

St. Sugar Canine Cancer Detection Training Center

Masao Miyashita

Nippon Medical School and Twin Peaks Laboratory of Medicine

\title{
Breast Cancer Detection from a Urine Sample by Dog Sniffing
}

\section{Research article}

Keywords: dogs, diagnosis, canine cancer detection, breast cancer, urine sample

Posted Date: October 14th, 2020

DOI: https://doi.org/10.21203/rs.3.rs-89484/v1

License: (c) (1) This work is licensed under a Creative Commons Attribution 4.0 International License. Read Full License 


\section{Abstract}

Background: Breast cancer is a leading cause of cancer death worldwide. Several studies have demonstrated that dog can sniff and detect cancer in the breath or urine sample of a patient.

Objective: The aim of this study is to assess whether the trained dog can detect breast cancer from urine samples.

Methods: A nine-year-old female Labrador Retriever was trained to identify cancer from urine samples of breast cancer patients. Urine samples from patients histologically diagnosed with primary breast cancer, those with non-breast malignant diseases, and healthy volunteers were obtained, and a double-blind test was performed.

Results: 40 patients with breast cancer, 142 patients with non-breast malignant diseases, and 18 healthy volunteers were enrolled, and their urine samples were collected. In 40 times out of 40 runs of a double-blind test, the trained dog could correctly identify urine samples of breast cancer patients. Sensitivity and specificity of this breast cancer detection method using dog sniffing were both $100 \%$.

Conclusions: The trained dog in this study could accurately detect breast cancer from urine samples of breast cancer patients. These results indicate the feasibility of a method to detect breast cancer from urine samples using dog sniffing in the diagnosis of breast cancer.

\section{Introduction}

Breast cancer was considered a major health issue among women, and is the second most common cause of cancer death throughout the world [1]. Early detection of the breast cancer is important for more efficient treatment. Currently, mammography (MG) is the most commonly used screening test, and has a reported sensitivity and specificity of $77 \%$ and $91 \%$, respectively [2]. Although breast cancers can be detected during the asymptomatic phase and reduces mortality among women of certain ages [3-5], MG still has several challenges. First, prevalence of MG is not sufficiently utilized even in developed countries. The rate of check-ups for women aged $65-74$ years is $16-20 \%$ and $43-46 \%$ for women aged $40-54$ years in Japan [6]. Second, non-malignant lesions are also detected, which sometimes leads to unnecessary testing, treatment, and anxiety [7], and at the same time, MG is less sensitive in dense breast [8]. Furthermore, mortality reduction in women ages $<40$ years has not yet been proven. Third, MG is associated with significant pain due to the relatively strong pressure applied to the breast. Fourth, there is the risk of radiation exposure especially in younger women with abnormal germline genes [9]. Given above drawbacks of MG, an alternative test with better compliance is needed to detect breast cancer in an early stage.

Cancer detection by dog sniffing (hereinafter referred to as "canine cancer detection") is one of the candidates as a new method to detect breast cancer. Detection threshold has been shown to be as low as 1.5 parts per trillion (ppt) [10]. Trained dogs can successfully discriminate between patients with cancers of skin [11, 12], bladder [13], lung [14-18], breast [14, 19, 20], prostate [20-22] ovary [23-25], colorectal [19, 26], liver [27], uterine cervix [28] from controls on the basis of odors in breath, urine, blood or cell culture medium. However, the canine cancer detection testing for breast cancer using human urine samples has not yet been attempted. We hypothesized that scent-trained dogs can discriminate between breast cancer patients and non-breast cancer patients using urine samples. The aim of this study was to investigate the efficacy of a canine cancer detection method using urine samples of patients diagnosed with breast cancer.

\section{Materials And Methods Patients and controls}

Patients with primary breast cancer, patients with non-breast malignant diseases, and healthy control volunteers at Nippon Medical School Chiba-Hokusoh Hospital and the Jizankai Medical Foundation Tsuboi Cancer Center Hospital from January 2011 to October 2012 were enrolled. Diagnosis was based on clinical assessment using MG and/or ultrasound and confirmed preoperatively by histological examination of core needle biopsy (CNB) samples. Patients who received a surgical operation before urine sample collection, and those with other types of cancer were excluded. Patients with non-breast malignant diseases were confirmed by biopsy. For female patients, MG and/or ultrasound was performed to rule out breast cancers. Healthy volunteers were verified with systematic cancer screening tests including blood test, chest X-ray, abdominal ultrasound, MG, and gynecological examination.

\section{Urine sampling}

Urine samples of the participants were collected with paper cups (Harn cup laminate A, Nissho Sangyo, Tokyo, Japan), and transferred to sterile test tubes (Sterile SP tube, Eiken Chemical Co., Tokyo, Japan) and each test tube was sealed with a cap. Urine samples of the breast cancer patients were collected a few days prior to surgery. The test tube samples were then stored at $-20^{\circ} \mathrm{C}$ until $1 \mathrm{ml}$ of the selected samples was used for the dog sniffing test.

\section{Dog and training}

A nine-year-old female Labrador Retriever was provided by the St. Sugar Canine Cancer Detection Training Center in Minamiboso City, Chiba, Japan [26]. The training was performed by a professional dog trainer with a similar procedure described in the previous report [26]. Briefly, each cancer detection training session was considered complete when the dog correctly detected breath samples from a cancer patient and four controls in dozens of trials. The correct behavior was rewarded by simultaneous play with a tennis ball. Training consisted of following steps: In the first step, the dog was trained to detect a breast cancer breath sample from five breath-sampling bags with the end caps on, which included three healthy and one non-breast malignant disease breath 
samples. As the dog successfully accomplished this task, the healthy controls were gradually replaced with non-breast malignant disease breath samples. In the final step, the dog was trained to detect a breast cancer sample from among five samples which included four non-breast malignant disease breath samples. This could subsequently be applied to urine samples. In this way, the dog was trained to be able to detect breast cancer patients. Under certain conditions, the dog sniffing test could not be conducted because the dog could not maintain concentration. These included weather conditions such as high temperature and high humidity in the summer.

\section{Detection of urine samples from breast cancer patients}

The test boxes were wooden, storage containers $27 \times 30 \times 20 \mathrm{~cm}$ in size. Each box is equipped with a $10 \mathrm{~cm}$ wall inside to hold a urine sample tube. So the dog does not come in direct contact with the test sample, each box was covered with a metal mesh. To conduct the test, 5 test boxes were placed in a straight line on the floor, one meter apart (Fig. 1). The sample tubes were kept separate to avoid any possibility of contamination of the control samples with volatile chemicals from the cancer samples. Double-blind testing, blinded to the dog trainer and the dog, was performed using one breast cancer sample and 4 control samples per test-run. Correct responses included (1) sitting down in front of a sample box containing a urine sample from patients with breast cancer (true positive in sensitivity calculations) and (2) only sniffing the odor of a sample and not sitting in front of a control sample (true negative). Incorrect responses included (1) sitting in front of a control sample (false positive) and (2) not sitting in front of a sample from a patient with breast cancer (false negative). Once the dog signaled in on a sample, it was given a reward. In each testing, the concentration level (high or low) of the dog was assessed and recorded. No adverse events, injury or illness to the dog was observed.

\section{Statistical analysis}

The Kruskal-Wallis test was used to analyze the clinical characteristics of the patients and controls. The percentage of correct detection per session was calculated for each test-run. Diagnostic accuracy was calculated as the sensitivity and specificity of the dog's identification of positive urine samples compared to the histopathological diagnosis of breast cancer. Thus, sensitivity of the test is the proportion of cancer samples correctly identified by the dog while specificity is the proportion of control samples negatively indicated by the dog. A P value $<0.05$ was considered statistically significant. All statistical analyses were completed using SPSS v.25 (IBM Corp., Armonk, NY, USA).

\section{Results \\ Patients}

A total of 200 participants were randomly selected in the study, and included 40 patients with primary breast cancer, 142 patients with non-breast malignancies, and 18 healthy individuals. All breast cancer patients were female. Histological diagnoses were ductal carcinoma in situ (six cases), nonspecified invasive ductal carcinoma/ invasive carcinoma (33 cases), and mucinous carcinoma (one case). Pathological stages of the breast cancer patients were classified according to the Union for International Union Cancer Control (UICC) classification as follows: Stage 0 (ductal carcinoma in situ) for 6 cases, Stage I for 19 cases, Stage IIA for 13 cases, and Stage IIIB for 2 cases). One patient with invasive ductal carcinoma, Stage IIIB had preoperative chemotherapy before the operation and collecting a urine sample. Non-breast malignancy patients are listed in Table 1.

Table 1

Number of non-breast malignancy cases and healthy volunteers used as controls. 


\begin{tabular}{ll}
\hline Diagnosis & Number \\
\hline Gastric cancer & $38(26.0 \%)$ \\
Cervical cancer & $36(24.7 \%)$ \\
HSIL & $21(14.4 \%)$ \\
Endometrial cancer & $17(12 \%)$ \\
Ovarian cancer & $16(11.0 \%)$ \\
Colorectal cancer & $7(4.8 \%)$ \\
Peritoneal cancer & $3(2.0 \%)$ \\
Uterine sarcoma & $2(1.4 \%)$ \\
Esophageal cancer & $1(0.7 \%)$ \\
Endometrial stromal sarcoma & $1(0.7 \%)$ \\
Vulvar cancer & $1(0.7 \%)$ \\
Liposarcoma & $1(0.7 \%)$ \\
Metastatic adrenal carcinoma & $1(0.7 \%)$ \\
LSIL & $1(0.7 \%)$ \\
Total & 146 \\
\hline
\end{tabular}

HSIL, high-grade squamous intraepithelial lesion; LSIL, low-grade squamous intraepithelial lesion

Median ages of breast cancer patients, non-breast malignancy patients, and healthy individuals were 57.5 (range 28-84), 57 (range 18-88) and 52 (range 2766) years old, respectively. Age distribution did not significantly vary among the patients and controls $(p=0.087)$.

\section{Dog condition and round times before decision}


Sample combinations of each test-run. Diagnosis/healthy, age, and stage are listed. Stages indicate pathological stage according to UICC classification.

Box 1

Box 2

Test- Diagnosis Age Stage Diagnosis Age Stage Diagnosis

run

$\begin{array}{llllllllllllllllllll}1 & \text { HSIL } & 31 & \text { NA } & \text { GC } & 41 & \text { IB } & \text { HSIL } & 36 & \text { NA } & \text { BC } & & 33 & 0 & \text { HSIL } & 35 & \text { NA }\end{array}$

$2 \quad$ EmC $\quad 71 \quad$ IB $\quad$ GC $\quad 77 \quad$ IA $\quad$ BC

$\begin{array}{llllllllll}3 & \text { GC } & 60 & \text { IB } & \text { CC } & 51 & \text { IIB } & \text { CC }\end{array}$

$\begin{array}{lllllllllll}4 & \mathrm{CC} & 85 & \text { IIIB } & \text { CRC } & 71 & \text { IIIB } & \text { CRC } & 68 & \text { IIIB } & \text { BC }\end{array}$

$5 \quad$ GC $\quad 74 \quad I B \quad$ EC

$\begin{array}{lllllll}6 & \text { CRC } & 70 & \text { I } & \text { EmC } & 70 & \text { IIIC }\end{array}$
Box 4

Age Stage Diagnosis

GC

CRC

BC

HSIL

$\begin{array}{llll}\text { Metastatic } & 80 & \text { IV } & \text { CC }\end{array}$

adrenal

cancer
Box 5

Diagnosis Age Sta!

$\begin{array}{lll}\text { BC } & 74 & \text { I } \\ \text { CC } & 53 & \text { IIB } \\ \text { CRC } & 68 & \text { IIIB } \\ \text { HSIL } & 50 & \text { NA } \\ \text { BC } & 75 & \text { । }\end{array}$

$7 \quad$ OC $\quad 51 \quad$ IIIC $\quad$ GC

$8 \quad$ GC $\quad 57 \quad$ IA $\quad$ OC $\square$ rec $\square \quad 58 \quad$ NA $\quad$ PerC

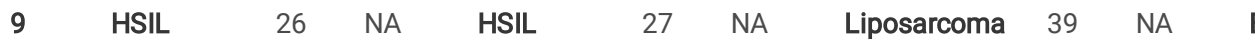

10 HSIL $\quad 44$ NA $\quad$ US $\quad 41$ IV $\quad$ GC

11 HSIL $\quad 44$ NA $\quad$ ESS $\quad 41 \quad$ IV $\quad$ GC

$\begin{array}{llllllll}12 & \text { EmC } & 70 & \text { IB } & \text { GC } & 71 & \text { IA } & \text { CC }\end{array}$

$\begin{array}{llllllll}13 & \text { HSIL } & 30 & \text { NA } & \text { HSIL } & 36 & \text { NA } & \text { B }\end{array}$

$14 \quad \mathrm{CC} \quad 61 \quad \mathrm{IB} \quad \mathrm{OC}(\mathrm{rec} \square \quad 65 \quad \mathrm{NA} \quad \mathrm{BC}$

$\begin{array}{lllllll}15 & \text { GC } & 64 & \text { IA } & \text { BC } & 59 & \text { I }\end{array}$

16 HSIL 39 NA $\quad 3$ LSIL $44 \quad$ NA

$\begin{array}{llllllll}17 & \text { HSIL } & 18 & \text { NA } & \text { CC } & 82 & \text { IIIB } & \text { BC }\end{array}$

18 HSIL $\quad 44 \quad$ NA $\quad$ BC

19 OCDrec $56 \quad$ NA $\quad$ CC $\quad 57 \quad$ IA $\quad$ OC

$\begin{array}{lllllllll}20 & \text { BC } & 67 & \text { IIA } & \text { CC } & 65 & \text { IV } & \text { CC }\end{array}$

cancer

22 healthy 39 NA $\quad$ EmC $49 \quad$ IB $\quad$ BC

$\begin{array}{llllllll}23 & \text { GC } & 64 & \text { IA } & \text { BC } & 64 & \text { । } & \text { CC }\end{array}$

24 Healthy $35 \quad$ NA GC

25 OC $\quad 50 \quad$ IC $\quad$ Healthy $39 \quad$ NA $\quad$ GC

26 Healthy 78 NA $\quad$ CC $\quad 34 \quad$ IA $\quad$ HSIL

27 Healthy $56 \quad$ NA $\quad$ GC $\quad 77 \quad$ IA $\quad$ BC

$\begin{array}{lllllllllllll}28 & \mathrm{CC} & 51 & \mathrm{llb} & \mathrm{GC} & 51 & \mathrm{IA} & \mathrm{CC}\end{array}$

29 GC $\quad 70 \quad$ IA $\quad$ EmC $\quad 70 \quad$ IIIC

$30 \quad C$

$31 \quad$ EmC $\quad 71 \quad$ IB

$\begin{array}{llllll} & 51 & N A & E m C & 70 & \text { IB }\end{array}$

$\begin{array}{lllllllllll}33 & \text { CC } & 62 & \text { IB } & \text { OC } & 61 & \text { IA } & \text { Healthy } & 53 & \text { NA }\end{array}$

34 Healthy $50 \quad$ NA BC

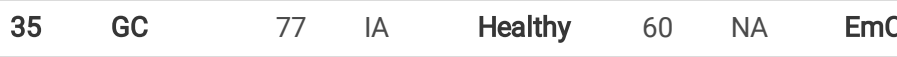

$\begin{array}{llll}36 & \text { BC } & 59 & \text { I }\end{array}$

$37 \quad$ GC

$57 \quad$ IIA

38 Healthy $56 \quad$ NA

$\begin{array}{lll}\text { CC } & 61 & \text { IB } \\ \text { Healthy } & 57 & \text { NA }\end{array}$

CRC
$\begin{array}{llll}B C & 49 & \text { IIA } & \text { HSIL }\end{array}$

GC

BC

CC

$\mathrm{CC}$

$\mathrm{BC}$

GC

EmC

CC

BC

cc

cc

cc

$\mathrm{CC}$

BC

GC

Healthy

CC

BC

BC

GC

$\begin{array}{llll}C & 47 & \mathrm{ClS} & \mathrm{BC}\end{array}$

Healthy

Uterine

sarcoma

OC

ocrecl

Emc

Healthy

CC

BC

Page 5/11

GC

\begin{tabular}{lllll}
70 & IA & GC & 70 & IA \\
\hline 63 & II & BC & 56 & IIA \\
\hline 64 & IIIB & EmC & 57 & IIIA \\
47 & NA & BC & 60 & 0 \\
80 & IV & CC & 85 & IIIB \\
& & & &
\end{tabular}

46 NA EmC 44 IB

$\begin{array}{lllll}60 & \text { IIA } & \text { BC } & 62 & \text { IIIB }\end{array}$

$\begin{array}{lllll}28 & \text { I } & \text { GC } & 41 & \text { IB }\end{array}$

54 IIIB BC $50 \quad 0$

54 IIIB $\quad$ BC $50 \quad 0$

69 I $\quad$ CCDrec $\quad 69$ NA

$\begin{array}{lllll}55 & \text { IV } & \text { CC } & 34 & \text { IA }\end{array}$

$\begin{array}{lllll}79 & \text { IV } & \text { GC } & 75 & \text { IA }\end{array}$

57 IA PerC (rec) 65 NA

$\begin{array}{llll}47 & \text { IIA HSIL } & 39 & \text { NA }\end{array}$

36 IA HSIL 38 NA

$\begin{array}{lllll}41 & \text { IA } & \text { CC } & 44 & \text { IIIB }\end{array}$

$\begin{array}{lllll}61 & \text { IB } & \text { BC } & 59\end{array}$

$\begin{array}{lllll}62 & \text { IB } & \text { GC } & 63 & \text { IA }\end{array}$

$\begin{array}{lllll}84 & \text { I } & \text { GC } & 77 & \text { IA }\end{array}$

$\begin{array}{lllll}46 & \text { IA } & \text { HSIL } & 42 & \text { NA }\end{array}$

$\begin{array}{lllll}27 & N A & O C & 61 & \text { IA }\end{array}$

$38 \quad$ IIIB $\quad$ BC $\quad 46 \quad$ ।

$\begin{array}{lllll}48 & \text { I } & \text { GC } & 55 & \text { IA }\end{array}$

$\begin{array}{lllll}34 & \text { I HSIL } & 35 & \text { NA }\end{array}$

$\begin{array}{lllll}71 & \text { IA } & \text { CRC } & 70 & \text { IV }\end{array}$

49 IIA EmC $49 \quad$ IB

$\begin{array}{llllll}\text { CRC } & 68 & \text { IIIB } & \text { BC } & 69 & \text { IIA }\end{array}$

$\begin{array}{llllll}\text { BC } & 44 & \text { IIA } & \text { CC } & 42 & \text { IIIb }\end{array}$

Healthy $\quad 66 \quad$ NA $\quad$ OC $\quad 85 \quad$ IIIC

GC $\quad 70 \quad$ IA $\quad$ EmC 63 IC

$\begin{array}{llllll}\text { BC } & 61 & \text { I } & \text { GC } & 62 & \text { IA }\end{array}$

$\begin{array}{lllllll}\text { GC } & 55 & \text { IA } & \text { CC } & 57 & \text { IA }\end{array}$

$\begin{array}{llllll}\text { GC } & 75 & \text { IA } & \text { BC } & 75 & \text { । }\end{array}$

$\begin{array}{llllll}\text { EmCIrec } & 58 & \text { NA } & \text { GC } & 57 & \text { IA }\end{array}$

$\begin{array}{llllll}\text { CC } & 51 & \text { IIB } & \text { BC } & 56 & \text { IIA }\end{array}$

$60 \quad$ IIA CC

$53 \quad$ IIA 


\begin{tabular}{|llllllllllllllllll}
39 & Healthy & 45 & NA & OC & 49 & IA & GC & 41 & IB & BC & 45 & I & EmC & 49 & IB \\
\hline 40 & GC & 64 & IA & OC & 68 & IA & BC & 64 & I & OC & 56 & IA & Healthy & 60 & NA \\
\hline
\end{tabular}

HSIL, high-grade squamous intraepithelial lesion; NA, not applicable; GC, gastric cancer; BC, breast cancer; EmC, Endometrial cancer; CC, cervical cancer; CRC, colorectal cancer; EC, esophageal cancer; (rec), recurrence; PerC, peritoneal cancer; US, uterine sarcoma; ESS, endometrial stromal carcinoma; OC, ovarian cancer; LSIL, low-grade squamous intraepithelial lesion; rec, recurrence; US, uterine sarcoma.

In 4 out of 40 times, the dog's concentration level was low, and the remaining runs were normal. The dog's low concentration were noted for 2 days, when testrun numbers 18-22, which were the hottest days in July, were performed. On these days, the room temperature was $26.3^{\circ} \mathrm{C}$ to $26.8^{\circ} \mathrm{C}$, and humidity was $83 \%$.

The round times before the dog's response ranged between one to three times. In further detail, the dog made one round in 14 runs, two rounds in 19 runs, and three rounds in seven runs.

\section{Sensitivity and specificity of the detection test}

Comparison of the cancer determination by dog sniffing versus pathological diagnosis among cancer patients and controls was calculated. The dog detected the breast cancer samples correctly in all test-runs (40/40). Thus, among the breast cancer patients and controls, overall sensitivity and specificity were both $100 \%$.

\section{Discussion}

The novelty of this study was to investigate the feasibility and efficacy of using the trained dog to detect and distinguish urine samples of breast cancer patients from a control group comprised of a variety of other malignancies and healthy volunteers. Using our established training method, the dog could be trained to detect breast cancer from urine samples, and $100 \%$ sensitivity and specificity rates could be achieved in the double-blind test series. Up to now, efficacy of urine samples has not been well clarified. Using urine samples is useful because of its simplicity and non-invasiveness. Some trained dogs were reported to discriminate between the urine of patients with urinary tract and prostate cancers from those of controls [13,21], but this is the first study indicating the efficacy and accuracy of a dog trained to detect breast cancer from urine samples.

Originally, in 1989, the hypothesis that dog can smell a cancer odor was raised during the consultation with a woman who claimed to have sought medical help as a direct result of her dog's inordinate interest in a skin lesion, which subsequently proved to be a malignant melanoma [29]. A similar case of patientdog interactions leading to cancer diagnoses was subsequently reported, suggesting the possibility of the existence of a cancer specific odor [30]. Initially, these "anecdotal" events were not supported by evidence. However, the following studies have demonstrated canine cancer detection for cancer screening is promising, feasible, and safe (Table 3) [11-28].

Table 3

Published reports on canine detection of various cancer types. 


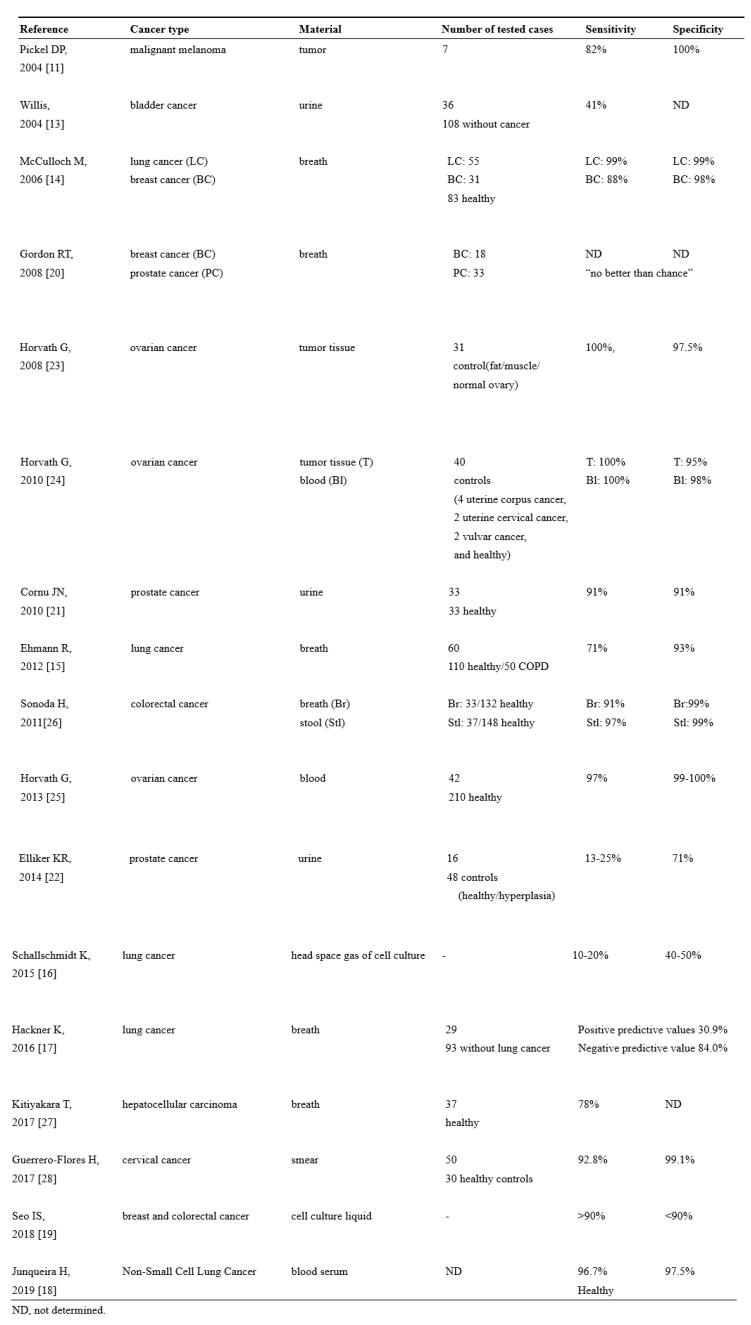

McCulloch et al. reported that trained dogs could successfully detect breast cancers using exhaled breath samples [14]. Breath samples from 31 breast cancer patients and healthy control patients were used, and sensitivity and specificity were 0.88 and 0.98 , respectively, across all stages. Sonoda et al. further investigated the utility of canine cancer detection in CRC using breath and watery stool [26]. Sensitivity and specificity in comparison with diagnosis by colonoscopy were 0.91 to 0.97 , and 0.99 , respectively. In order to determine whether a specific cancer odor does exist, or a particular natural scent disappears due to the cancer, a mixture of watery stool of CRC cancer patients and controls was produced, and the sample could be correctly identified by the dog. From this, it was surmised that chemical compounds from cancer could be circulating throughout the body. Next, focus was placed on whether these odors were cancer-common or cancer-specific. In several subsequent series, when one type of cancer sample was used as the standard scent, the dog was able to differentiate between other types of cancers [26,31]. Seo et al. also reported that metabolic wastes of both breast and CRC in vitro have a common specific odor [19]. On the other hand, several types of cancers which were included as controls could be successfully identified as the targeted cancer by the sniffer dog, which is consistent with the results of this study [24]. These studies suggested that both of cancer-common scents and cancer-specific scents exist. Consequently, non-breast malignancies as well as healthy volunteers were included in the control group. The dog in this test successfully differentiated breast cancer from non-breast malignancies and healthy controls, and this concurs with previous studies [24].

The test data at this time showed a higher sensitivity and specificity compared to other previous reports. One possible reason is the environmental settings of the test-run, which allowed the dog to respond without stress. Tests were not carried out under the stressful conditions for the dog. Detection accuracy may be influenced by the condition of the dog, and therefore performance should be systematically monitored [32]. In addition, storage of the sample until testing may be pivotal in maximizing detection rate. While the effect of storage duration or method on detection accuracy was not assessed in this study, standardization of such methodology, in order to utilize this approach for a mass screening, should be considered [20].

Evidence has shown that human body emits a wide array of volatile organic compounds (VOCs), both odorous and non-odorous, depending on individual background [33]. These VOCs are emitted throughout the body, including breath, blood and urine [34, 35]. According to analysis of VOCs, different volatile patterns have been correlated with a variety of diseases including cancers [14,33,36,37], which dogs can be trained to detect. Consequently, analysis of cancer specific VOCs is considered feasible. Some studies have attempted to demonstrate cancer specific VOCs by utilizing gas chromatography-mass spectrometry (GCMS) [38]. The potential of VOCs in urine, breath and blood samples to be biomarkers for an array of diseases could be demonstrated [35, 39]. However, VOCs are affected by physiological factors such as dietary and smoking habits, infections, and benign diseases [40], which GCMS cannot detect all or even nearly all chemicals present [14], nor clarify the exact chemical compounds and/or their combinations. Combining this dog-based study with instrument-based research would be mutually beneficial for further analysis [41]. 
This study has limitations. Our cancer detection system relies on one trained dog. Expanding this to multiple training centers with expert trainers, multiple dogs, and over several years is desired. An effective training protocol is essential for good performance [20]. Even though dogs can be subject to the same training manner by the same trainer, inter-dog difference or poor performance due to stress have been pointed out in similar studies [10,17].

\section{Conclusions}

In conclusion, this study represents a novel attempt to use cancer detection dogs to distinguish between breast cancer and non-breast malignancy and healthy controls from urine samples. This method was demonstrated to be more accurate and less invasive than existing screening methods.

\section{List Of Abbreviations}

CNB: Core needle biopsy

CRC: Colorectal cancer

GCMS: Gas chromatography-mass spectrometry

HSIL: High-grade squamous intraepithelial lesion

LSIL: Low-grade squamous intraepithelial lesion

MG: Mammography

UICC: Union for International Cancer Control

VOC: Volatile organic compounds

\section{Declarations}

\section{Ethics approval and consent to participate}

Participants voluntarily enrolled in this study and provided written informed consent. This study was conducted in accordance with the principles embodied in the Declaration of Helsinki, and was approved by the ethics committees of Nippon Medical School Chiba Hokusoh Hospital (IRB\#320).

\section{Consent for publication}

Not applicable.

\section{Availability of data and materials}

The datasets used and/or analyzed during the current study are available from the corresponding author on reasonable request.

\section{Competing interests}

The authors declare that they have no competing interests.

\section{Funding}

This study is funded by Research Grants of Department of Surgery, Nippon Medical School Chiba Hokusoh Hospital.

\section{Authors' contributions}

SK, SI, MY, HT, NY, YS, and MM conceptualized and designed the study. SK, SI, MY, HT, NY, YS, and MM developed the methodology. SK, SI, MY, HT, NY, YS, and MM performed the data acquisition. SK, SI, MY, HT, NY, YS, and MM analyzed and interpreted the data. SK, SI, and MM wrote the manuscript, which was edited by all the authors who approved the final version. MM provided study supervision. All authors are guarantors of the integrity of the data collection and interpretation.

\section{References}

1. Ferlay J, Colombet M, Soerjomataram I, Mathers C, Parkin DM, Pineros M, Znaor A, Bray F: Estimating the global cancer incidence and mortality in 2018: GLOBOCAN sources and methods. Int J Cancer 2019, 144(8):1941-1953. 
2. Ohuchi N, Suzuki A, Sobue T, Kawai M, Yamamoto S, Zheng Y-F, Shiono YN, Saito H, Kuriyama S, Tohno E et al: Sensitivity and specificity of mammography and adjunctive ultrasonography to screen for breast cancer in the Japan Strategic Anti-cancer Randomized Trial (J-START): a randomised controlled trial. The Lancet 2016, 387(10016):341-348.

3. Nelson HD, Tyne K, Naik A, Bougatsos C, Chan BK, Humphrey L, Force USPST: Screening for breast cancer: an update for the U.S. Preventive Services Task Force. Ann Intern Med 2009, 151(10):727-737, W237-742.

4. Njor S, Nystrom L, Moss S, Paci E, Broeders M, Segnan N, Lynge E, Euroscreen Working G: Breast cancer mortality in mammographic screening in Europe: a review of incidence-based mortality studies. J Med Screen 2012, 19 Suppl 1:33-41.

5. Autier P, Boniol M: Mammography screening: A major issue in medicine. Eur J Cancer 2018, 90:34-62.

6. Uchida K, Ohashi H, Kinoshita S, Nogi H, Kato K, Toriumi Y, Yamashita A, Kamio M, Mimoto R, Takeyama H: Breast cancer screening and the changing population pyramid of Japan. Breast cancer (Tokyo, Japan) 2015, 22(2):172-176.

7. Brodersen J, Siersma VD: Long-term psychosocial consequences of false-positive screening mammography. Ann Fam Med 2013, 11(2):106-115.

8. Tohno E, Ueno E, Watanabe H: Ultrasound screening of breast cancer. Breast cancer (Tokyo, Japan) 2009, 16(1):18-22.

9. Warner E: Screening BRCA1 and BRCA2 Mutation Carriers for Breast Cancer. Cancers (Basel) 2018, 10(12):477-487.

10. Concha AR, Guest CM, Harris R, Pike TW, Feugier A, Zulch H, Mills DS: Canine Olfactory Thresholds to Amyl Acetate in a Biomedical Detection Scenario. Front Vet Sci 2018, 5:345.

11. Pickel D, Manucy GP, Walker DB, Hall SB, Walker JC: Evidence for canine olfactory detection of melanoma. Applied Animal Behaviour Science 2004, 89(12):107-116.

12. Elliker KR, Williams HC: Detection of skin cancer odours using dogs: a step forward in melanoma detection training and research methodologies. The British journal of dermatology 2016, 175(5):851-852.

13. Willis CM: Olfactory detection of human bladder cancer by dogs: proof of principle study. BMJ British medical journal (Clinical research ed) 2004, 329(7468):712.

14. McCulloch M, Jezierski T, Broffman M, Hubbard A, Turner K, Janecki T: Diagnostic accuracy of canine scent detection in early- and late-stage lung and breast cancers. Integrative cancer therapies 2006, 5(1):30-39.

15. Ehmann R, Boedeker E, Friedrich U, Sagert J, Dippon J, Friedel G, Walles T: Canine scent detection in the diagnosis of lung cancer: revisiting a puzzling phenomenon. The European respiratory journal 2012, 39(3):669-676.

16. Schallschmidt K, Becker R, Zwaka H, Menzel R, Johnen D, Fischer-Tenhagen C, Rolff J, Nehls I: In vitro cultured lung cancer cells are not suitable for animal-based breath biomarker detection. J Breath Res 2015, 9(2):027103.

17. Hackner K, Errhalt P, Mueller MR, Speiser M, Marzluf BA, Schulheim A, Schenk P, Bilek J, Doll T: Canine scent detection for the diagnosis of lung cancer in a screening-like situation. J Breath Res 2016, 10(4):046003.

18. Junqueira H, Quinn TA, Biringer R, Hussein M, Smeriglio C, Barrueto L, Finizio J, Huang XYM: Accuracy of Canine Scent Detection of Non-Small Cell Lung Cancer in Blood Serum. The Journal of the American Osteopathic Association 2019.

19. Seo IS, Lee HG, Koo B, Koh CS, Park HY, Im C, Shin HC: Cross detection for odor of metabolic waste between breast and colorectal cancer using canine olfaction. PLoS One 2018, 13(2):e0192629.

20. Gordon RT, Schatz CB, Myers LJ, Kosty M, Gonczy C, Kroener J, Tran M, Kurtzhals P, Heath S, Koziol JA et al: The use of canines in the detection of human cancers. Journal of alternative and complementary medicine (New York, NY) 2008, 14(1):61-67.

21. Cornu JN, Cancel-Tassin G, Ondet V, Girardet C, Cussenot O: Olfactory detection of prostate cancer by dogs sniffing urine: a step forward in early diagnosis. European urology 2011, 59(2):197-201.

22. Elliker KR, Sommerville BA, Broom DM, Neal DE, Armstrong S, Williams HC: Key considerations for the experimental training and evaluation of cancer odour detection dogs: lessons learnt from a double-blind, controlled trial of prostate cancer detection. BMC urology 2014, 14:22.

23. Horvath G, Jarverud GA, Jarverud S, Horvath I: Human ovarian carcinomas detected by specific odor. Integrative cancer therapies 2008, 7(2):76-80.

24. Horvath G, Andersson H, Paulsson G: Characteristic odour in the blood reveals ovarian carcinoma. BMC cancer 2010, 10:643.

25. Horvath G, Andersson H, Nemes S: Cancer odor in the blood of ovarian cancer patients: a retrospective study of detection by dogs during treatment, 3 and 6 months afterward. BMC cancer 2013, 13:396.

26. Sonoda H, Kohnoe S, Yamazato T, Satoh Y, Morizono G, Shikata K, Morita M, Watanabe A, Morita M, Kakeji Y et al: Colorectal cancer screening with odour material by canine scent detection. Gut 2011, 60(6):814-819.

27. Kitiyakara T, Redmond S, Unwanatham N, Rattanasiri S, Thakkinstian A, Tangtawee P, Mingphruedhi S, Sobhonslidsuk A, Intaraprasong P, Kositchaiwat C: The detection of hepatocellular carcinoma (HCC) from patients' breath using canine scent detection: a proof-of-concept study. J Breath Res 2017 , 11(4):046002.

28. Guerrero-Flores H, Apresa-García T, Garay-Villar Ó, Sánchez-Pérez A, Flores-Villegas D, Bandera-Calderón A, García-Palacios R, Rojas-Sánchez T, RomeroMorelos P, Sánchez-Albor V et al: A non-invasive tool for detecting cervical cancer odor by trained scent dogs. BMC cancer 2017, 17(1):79.

29. Williams H, Pembroke A: SNIFFER DOGS IN THE MELANOMA CLINIC? The Lancet 1989, 333(8640):734.

30. Church J, Williams H: Another sniffer dog for the clinic? The Lancet 2001, 358(9285):930.

31. McCulloch M, Turner K, Broffman M: Lung cancer detection by canine scent: will there be a lab in the lab? The European respiratory journal 2012, 39(3):511-512. 
32. Jezierski T, Walczak M, Ligor T, Rudnicka J, Buszewski B: Study of the art: canine olfaction used for cancer detection on the basis of breath odour. Perspectives and limitations. Journal of Breath Research 2015, 9(2):027001.

33. Shirasu M, Touhara K: The scent of disease: volatile organic compounds of the human body related to disease and disorder. Journal of biochemistry 2011, 150(3):257-266.

34. Monteiro M, Moreira N, Pinto J, Pires-Luis AS, Henrique R, Jeronimo C, Bastos ML, Gil AM, Carvalho M, Guedes de Pinho P: GC-MS metabolomics-based approach for the identification of a potential VOC-biomarker panel in the urine of renal cell carcinoma patients. J Cell Mol Med 2017, 21(9):2092-2105.

35. Woollam M, Teli M, Angarita-Rivera P, Liu S, Siegel AP, Yokota H, Agarwal M: Detection of Volatile Organic Compounds (VOCs) in Urine via Gas Chromatography-Mass Spectrometry QTOF to Differentiate Between Localized and Metastatic Models of Breast Cancer. Sci Rep 2019, 9(1):2526.

36. Phillips M, Cataneo RN, Ditkoff BA, Fisher P, Greenberg J, Gunawardena R, Kwon CS, Rahbari-Oskoui F, Wong C: Volatile markers of breast cancer in the breath. The breast journal 2003, 9(3):184-191.

37. Phillips M, Gleeson K, Hughes JM, Greenberg J, Cataneo RN, Baker L, McVay WP: Volatile organic compounds in breath as markers of lung cancer: a cross-sectional study. Lancet (London, England) 1999, 353(9168):1930-1933.

38. Phillips M, Bauer TL, Pass HI: A volatile biomarker in breath predicts lung cancer and pulmonary nodules. Journal of Breath Research 2019 , $13(3): 036013$.

39. Jobu K, Sun C, Yoshioka S, Yokota J, Onogawa M, Kawada C, Inoue K, Shuin T, Sendo T, Miyamura M: Metabolomics study on the biochemical profiles of odor elements in urine of human with bladder cancer. Biological \& pharmaceutical bulletin 2012, 35(4):639-642.

40. Fischer S, Trefz P, Bergmann A, Steffens M, Ziller M, Miekisch W, Schubert JS, Kohler H, Reinhold P: Physiological variability in volatile organic compounds (VOCs) in exhaled breath and released from faeces due to nutrition and somatic growth in a standardized caprine animal model. $J$ Breath Res 2015 , $9(2): 027108$.

41. Pleil J, Giese R: Integrating exhaled breath diagnostics by disease-sniffing dogs with instrumental laboratory analysis. J Breath Res 2017,11 (3):032001.

\section{Figures}
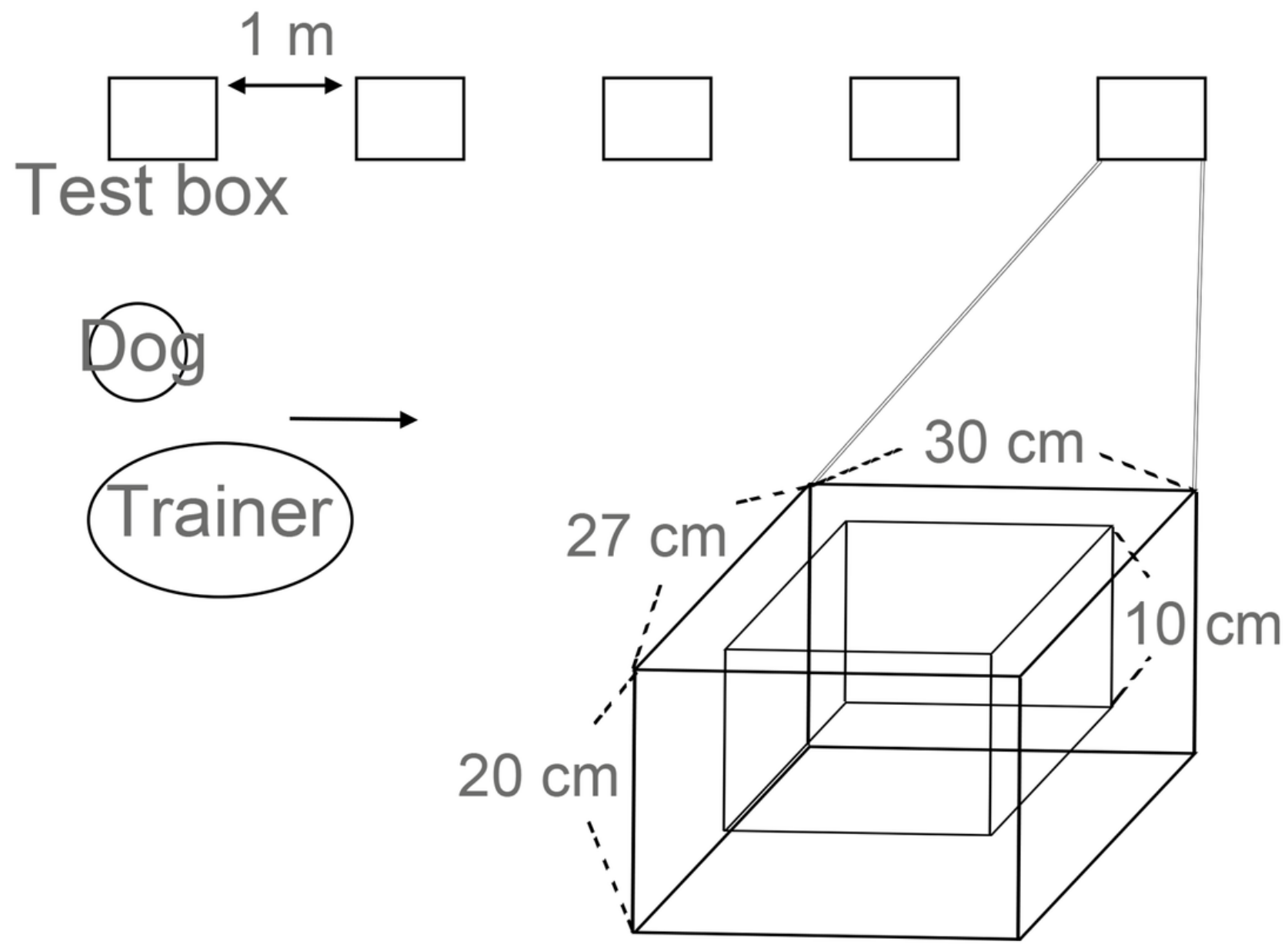

Figure 1 
Test design. The test boxes are wooden, storage containers $27 \times 30 \times 20 \mathrm{~cm}$ in size. Each box is equipped with a $10 \mathrm{~cm}$ deep wall inside to hold either a breath sample bag or a urine sample tube. The 5 test boxes were placed in a straight line on the floor at a distance of 1 meter apart. 